\title{
Follicular Dendritic Cell Sarcoma Mediastinum - a case report
}

\author{
Kirti Bushan
}

Received: 27 November 2012 / Accepted: 27 August 2014 / Published online: 4 September 2014

(C) Indian Association of Surgical Oncology 2014

\begin{abstract}
Follicular dendritic cell tumor (FDCT) are extremely rare difficult to diagnose category tumors. There has been a considerable controversy in medical community regarding precise classification and optimal management of this tumor with some treating it as a form of non Hodgkins lymphoma and some as soft tissue sarcomas. The number of published cases are still low and documentation too heterogenous to give statistically ified therapeutic recommendation of these tumors. This case report aims to highlight various aspects of diagnosing and treating this rare entity.
\end{abstract}

Keywords Follicular dendritic cell tumor $\cdot$ FDC markers

\section{Introduction}

A 37 years non-smoker, non-alcoholic gentleman with no medical comorbidities presented with complaints of low grade fever, weight loss and generalised malaise for 2 months. The clinical examination done by a physician outside revealed good performance status(PS 0 ECOG scale) with no clinical abnormality except for a palpable right supraclavicular lymph node. All routine blood tests including complete hemogram, renal and liver functions were normal. The chest $\mathrm{x}$-ray done revealed a soft tissue opacity without calcification with welldefined margins in right upper mid zone. He was advised CECT scan chest and neck which revealed a large mass lesion $8.9 \times 8.5 \times 8.0 \mathrm{~cm}$ in anterior superior mediastinum predominantly on right paratracheal region with peripherally enhancing solid and necrotic components; superiorly mass extending to thoracic inlet and inferiorly till carina. The trachea and right

K. Bushan $(\bowtie)$

Department of surgical oncology, Asian institute of oncology

Mumbai, Somaiya, Sion, Mumbai, Maharashtra 400022, India

e-mail: kirtibushan@gmail.com main bronchus were mildly compressed and pushed to left and anteriorly lesion reached upto the chest wall. The superior vena cava (SVC) was compressed and displaced superiorly and medially with the mass abutting right brachiocephalic artery and the aortic arch. There was no significant mediastinal or hillar adenopathy except for right supraclavicular lymph nodes were enlargement. Bone window, lung parenchyma, pleureal and pericardial spaces were normal. A CT guided tru-cut biopsy of mediastinal mass done outside revealed necrotic material with no well-defined granulomas. Tissue was ZNstain negative and PAS negative for AFB and fungal infection respectively. . Culture by TB MGIT 960 TB system revealed no growth of mycobacterium species. The palpable right supraclavicular lymph node $(3 \times 3 \times 1 \mathrm{~cm}$ size $)$ excision was done outside which revealed metastatic malignant tumor (undifferentiated carcinoma).

He was referred to a medical oncologist in our institute and was evaluated on the lines of malignancy of unknown origin (MUO). All the tumor markers including AFP and HCG were within normal limits. Repeat CT guided tru cut biopsy revealed follicular dendritic cell tumor with tumor expressing CD21, CD35, and CD23 (focally) and immuno negative for cytokeratin EMA. Careful clinical, radiological and pathologic assessment ruled out the possibility of germ cell tumor, Hodgkin's and Non-Hodgkin lymphoma or metastatic carcinoma. The patient was advised 3 cycles of chemo therapy followed by evaluation by medical oncologist. Three cycles of doxarubian and ifosfamide based chemotherapy was given at 3 weekly interval. Post chemotherapy CECT scan chest was done for response evaluation which showed progression of the disease. The mass lesion was $10.8 \times 10.3 \times 9.3 \mathrm{~cm}$ causing extrinsic compression of trachea and right main bronchus with narrowing of its lumen. There was compression and anterior displacement of superior vena cava with mass lesion abutting to proximal aortic arch, right innominate and subclavian vessels. Posteriorly the mass was reaching upto paravertebral 
space and causing extrinsic compression of right upper lobe bronchus as well. Clinical examination showed that patient had features of SVC compression with swelling of face and neck and mild dyspnoea on lying down.

He was referred to surgical oncology department for further management. The echocardiography and pulmonary functions were normal. He was optimised for surgery. Median sterenotomy was done. Mass was seen occupying retrosternal region with pre and paratracheal extension, abutting SVC, infiltrating right brachio cephalic vein and compressing right tracheo bronchial tree. A lot of post chemotherapy desmoplatic reaction was seen. Blunt and sharp dissection was done. Right brachiocephalic vein and azygous vein were ligated and right IVC was separated from the mass. Tracheo bronchial tree and right upper lung lobe were dissected away from the tumor mass. Whole mass was removed in it's entirety and R0 clearance was achieved. Post-operative period remained uneventful. Histopathology report revealed $11 \times 8$ $\times 7.5 \mathrm{~cm}$ capsulated well circumscribed tumor mass showing features of follicular dendritic cell sarcoma. On IHC, the tumor cells expressed vimentin, CD21, CD23, CD35 and EMA weakly. Centrally tumor was necrotic. The tumor capsule tissue showed tumor focally and thus patient was advised postoperative chemoradiation. The follow up period of last six months post completion of radiation therapy is uneventful.

\section{Discussion}

Follicular dendritic cell tumor (FDCT) are extremely rare difficult to diagnose category tumours. There has been a considerable controversy in medical community regarding precise classification and optimal management of this tumor with some treating it as a form of non Hodgkins lymphoma and some as soft tissue sarcomas. The number of published cases are still low and documentation too heterogeneous to give statiscally verified therapeutic recommendation for these tumours.

While the existence of FDCT was predicted by Lennert in 1978, the tumor wasn't recognised as its own cancer until 1986 after characterisation by Monda et al. [1, 2]. It accounts for $0.4 \%$ of all soft tissue tumor and is mostly diagnosed in young or middle aged adults. It is considered a low or intermediate grade malignancy but has significant recurrent or metastatic potential with low mortality [3]. Most cases develop in lymph node especially in cervical, axillary and mediastinal region but $30 \%$ cases develop in extra nodal sites which include head and neck ( palate, tonsil, pharynx, thyroid), breast, liver, spleen, pancreas and bowel [4-6]. Intra-abdominal FDCT tend to be more aggressive than FDCT involving nodes.

There is no definite cause for FDCT. There is however some evidence that previous exposure to Epstein Barr Virus (EBV) or hyaline vascular type Castleman's disease (HVCD) can increase risk. About 10-20\% of FDCTs are associated with Castleman's disease. Possible role of P53 overexpression in evolution of HVCD to PDTC has been suspected [7]. Symptoms largely depend on organ specific involvement. Most common symptons are painless swelling or mass, cough, sore throat, pressure symptoms, weight loss and tiredness.

Follicular differentiated cells (FDC's) are usually located in germinal centres of lymphoid follicles, functionally present as antigens to B-cells and thus improve quality of humoral immune response [8]. Accurate pathological diagnosis requires immunostaining with morphological, cytochemical and electron microscopic analyses. Grossly tumor is usually grey to tan with variable size. The neoplastic cells vary from spindle to oval with eosinophilic cytoplasm growing in sheets and forming fascicles or whorls reminiscent of those seen in meningiomas. The nuclei are oral or elongated with thin nuclear membrane, inconspicuous or small eosinophilic nucleoli and clear or dispersed chromatin. The tumor cells are characteristically admixed with small lymphocytes. The behaviour of these tumor is more in to low grade STS than a malignant lymphoma and is characterised by local recurrence and occasional metastasis. Tumors with aggressive behaviour show coagulative necrosis, marked cellular atypia, high mitotic rates (75 mitosis / $10 \mathrm{HPF)} \mathrm{and} \mathrm{abnormal} \mathrm{mitosis} \mathrm{[9,} \mathrm{10].}$

On immunohistochemical study with FDC markers, they are strongly positive for CD21, CD35, Ki-MP4, FDRC1P, clusterin. They are commonly positive for CD23, desmoplakin, NGFR, HLADR, vimentin, fascin. They are variably positive for EMA, S-100, CD68, and CD45. They are negative for CD1a, lysozyme, MPO, CD34, CD3, CD30, BCL2, SMA, AE1/3, Cam5.2 [11, 12]. Cellular mutations of FDC's have been exploited for diagnostic purpose. Characteristically FDCT have microtubuloreticular structure (MTRS) which helps in microtubule formation of many structures including mitotic spindle and cellular protein clusterin which helps in clearance of cellular debris and apoptosis [13]. Ultra structural analysis shows a complex inter-digitating cytoplasmic processes joined desmosomes (dendritic morphology). They show low level of lysosome enzymes and play key role in antigen presentation.

The key to successful management of FDCT largely depends on accurate and timely diagnosis. Complex surgical resection is the treatment of choice whenever feasible. In 2008, largest review of FDCT was published as a retrospective analysis of 98 patients who advocated curative surgery (R0 resection) as standard treatment with no adjuvant chemo radiation [14]. In case of R1 or R2 resections (residual disease), adverse pathologic features and recurrent disease, adjuvant chemotherapy and radiotherapy is recommended. Adjuvant chemo radiation after $\mathrm{R} 0$ resection has not shown any influence on disease free survival (DFS) but many studies have shown decrease in local recurrence rates after adjuvant chemo radiation is given in incompletely resected tumors [15]. 
To summarise, FDCT is a newly characterised cancer and with recent advances in molecular biology and better diagnostic assays, we hope major hurdle in treatment of FDCT. i.e., misdiagnosis is overcome and novel approaches to specially target and treat FDCT's will be developed.

\section{References}

1. Lennert K (1978) Malignant lymphomas other than Hodgkin's disease, histology, cytology, ultra structure, immunology. Springer, Berlin, pp 59-64

2. Monda L, Warnke R, Rosai J (1986) A primary lymph node malignancy with features suggestive of dendritic reticulum cell differentiation: A report of 4 cases. Am J Pathol 122(3):562-572

3. Sharpe M, Easthope SE, Keating GM, Lamb HM (2002) Polyethylene glycol - liposomal doxorubicin : a review of its use in the management of solid and haematological malignancies and AIDS related Kaposi's sarcoma. Drugs 62(14):2089-2126

4. WahCheu et al (2011) FDCT involving liver spleen and paripancreatic region. Am J Surg Pathol 25:721-731

5. Pruneri G, Masullo M, Renne G etal FDCS of breast : Virchow's Arch 2002 August; 441 : 194 - 99
6. Dominquez, Malagon H (2004) caro - Valderetal FDCT involving head and neck. Ann. Diagn Pathol 8:325-332

7. Chan AC (2001) Chan Kw, Chan JK etal Development of FDCS in HVCD of the nasopharynx : Tracing its evolution by sequential biopsies. Histopathology 38(6):510-518

8. Kosco MH, Gray D (1992) Signals involved in germinal centre reactions. Immunol Rev 126:63-76

9. Shiaj, Chen W, Tang LH, Carlson DL, QinjetalExtranodalFDCS : clinical, pathologic and histogenetic characteristics of an under recognised disease entity. VirchowsArchic 2006; 449 : 148 158.

10. Chan JK, Fletcher CD, Nayler SJ, Cooper K (1997) FDCS. Clinical pathologic analysis 17 cases suggesting a malignant potential higher than currently recognised. Caner 79:294-313

11. Granados R, Aramburu JA, Rodriguez JM (2008) Nieto MA cytopathology of a primary FDCS of the liver diagnosis cytopathology 36 : $42-46$

12. Marsee DK, Hornick JL et al (2009) Markers of FDC. Appl Immunohistochem Mol Morphol 19:102-107

13. Ono Y (2009) Terashima, Kazuo, Liu, Aimin, Yokoyamaetal FDCS with micro tubule reticular structure and virus like particle production vitro. Pathol Int 59:332-344

14. 14) Fonseca R, Yamakawa M, Nakamura Setal FDCS and interdigitatingreticulam cell sarcoma: a review AM J Hematol. $1998: 161-167$.

15. Perez, Ordonez B, Erlandson RA, Rosai J (1996) FDCT : report of 13 additional cases. Am J Surg Pathol 20:944-955 